\title{
Ligustrazine attenuates the platelet-derived growth factor-BB-induced proliferation and migration of vascular smooth muscle cells by interrupting extracellular signal-regulated kinase and P38 mitogen-activated protein kinase pathways
}

\author{
LIFEI YU*, XIAOJING HUANG* ${ }^{*}$ KAI HUANG, CHUN GUI, QIAOJUAN HUANG and BIN WEI \\ Department of Cardiology, Western Hospital, The First Affiliated Hospital of Guangxi Medical University, \\ Nanning, Guangxi 530021, P.R. China
}

Received March 23, 2014; Accepted January 2, 2015

DOI: $10.3892 / \mathrm{mmr} .2015 .3383$

\begin{abstract}
The abnormal proliferation and migration of vascular smooth muscle cells (VSMCs) leads to intimal thickening of the aorta and is, therefore, important in the development of arteriosclerosis. As a result, the use of antiproliferative and antimigratory agents for VSMCs offers promise for the treatment of vascular disorders. Although several studies have demonstrated that ligustrazine may be used to treat heart and blood vessel diseases, the detailed mechanism underlying its actions remain to be elucidated. In the present study, the inhibitory effect of ligustrazine on platelet-derived growth factor (PDGF)-BB-stimulated VSMC proliferation and migration, and the underlying mechanisms were investigated. The findings demonstrated that ligustrazine significantly inhibited PDGF-BB-stimulated VSMC proliferation. VSMCs dedifferentiated into a proliferative phenotype under PDGF-BB stimulation, which was effectively reversed by the administration of ligustrazine. In addition, ligustrazine also downregulated the production of nitric oxide and cyclic guanine monophosphate, induced by PDGF-BB. Additionally, ligustrazine significantly inhibited PDGF-BB-stimulated VSMC migration. Mechanistic investigation indicated that the upregulation of cell cycle-associated proteins and the activation of the extracellular signal-regulated kinase (ERK) and P38 mitogen-activated protein kinase (MAPK) signaling induced by PDGF-BB was suppressed by the administration of ligustrazine. In conclusion, the present study, demonstrated for the first time, to the best of our knowledge, that ligustrazine downregulated
\end{abstract}

Correspondence to: Professor Lifei Yu, Department of Cardiology, Western Hospital, The First Affiliated Hospital of Guangxi Medical University, 34 Daxue Road, Nanning, Guangxi 530021, P.R. China E-mail: guangxiyulifei@163.com

\section{"Contributed equally}

Key words: ligustrazine, vascular smooth muscle cell, proliferation, migration
PDGF-BB-induced VSMC proliferation and migration partly, at least, through inhibiting the activation of the ERK and P38 MAPK signaling.

\section{Introduction}

Abnormal proliferation and migration of vascular smooth muscle cells (VSMCs) causes intimal thickening of the aorta, and is, therefore, involved in the development of arteriosclerosis $(1,2)$. In addition, it may also lead to restenosis following percutaneous coronary intervention (PCI) or vein grafting (3). As a result, antiproliferative and antimigratory agents for VSMCs offer promise for the treatment of vascular disorders.

Ligustrazine, the main component of the Chinese medicine Rhizoma Chuanxiong, is important in the treatment of angiocardiopathy (4). Wang et al (5) demonstrated that ligustrazine had an antithrombotic effect on a model of induced arteriovenous shunt thrombosis; Luo et al (6) demonstrated that ligustrazine had a protective effect on hepatic energy metabolism in ischemia/reperfusion injury in the rat and Yu et al (7) revealed that ligustrazine protected the mitochondrial structure and the myocardial cell function in reversing angiotensin II-induced myocardial cell hypertrophy. However, the detailed role of ligustrazine in the regulation of VSMC proliferation and migration and the underlying molecular mechanism remains to be elucidated.

It is well-established that VSMCs remain in a quiescent state in physiological conditions and can switch to a markedly proliferative and migratory state in response to various stimuli, including inflammation and oxidative stress $(8,9)$. Platelet-derived growth factor (PDGF)-BB is important in vascular remodeling (10). Following vascular injury, PDGF-BB is markedly upregulated and activates proliferativeand migratory-associated signaling via binding to its receptor, PDGFR $\beta$, stimulating VSMC proliferation and migration (11). Nitric oxide (NO) has multiple protective effects on the cardiovascular system (12). The role of NO in the regulation of the vascular system is associated with a cyclic guanine monophosphate (cGMP)-dependent mechanism (13). However, whether ligustrazine has effects on endogenous $\mathrm{NO}$ production remains to be elucidated. 
The aim of the present study was to investigate the effect of ligustrazine on PDGF-BB-induced proliferation and migration in VSMCs and the underlying molecular mechanism. The findings demonstrated for the first time, to the best of our knowledge, that ligustrazine downregulated PDGF-BB-induced VSMC proliferation and migration, at least partially, through inhibiting the activation of the extracellular signal-regulated kinase (ERK) and P38 mitogen-activated protein kinase (MAPK) signaling.

\section{Materials and methods}

Materials and reagents. Ligustrazine was purchased from Guizhou Baite pharmacy (Guyang, China). Dulbecco's modified Eagle's medium (DMEM)/F12 medium and fetal bovine serum (FBS) were purchased from Life Technologies (Carlsbad, CA, USA). Dimethyl sulfoxide (DMSO) and MTT were purchased from Sigma-Aldrich (St. Louis, MO, USA). Recombinant human PDGF-BB was purchased from ProSpec-Tany TechnoGene (Rehovot, Israel). Mouse monoclonal anti-smooth muscle- $\alpha$-actin (SMA; 1:100; BM0002) antibodies were purchased from Boster Biological Technology, Ltd (Wuhan, China). Mouse monoclonal anti-desmin (1:100; sc-365130), anti-smoothelin (1:50; sc-376902), anti-phosphorylated (phosph)-ERK (1:100; sc-136521), anti-total ERK (1:50; sc-135900), anti-phospho-p38 (1:50; sc-81621), anti-total p38 (1:100; sc-7973), anti-cyclin-dependent kinase (CDK)2 (1:100; sc-6248), anti-CDK4 (1:50; sc-23896), anti-cyclin D1 (1:50; sc-450), anti-cyclin E (1:100; sc-48420) and anti-glyceraldehyde 3-phosphate dehydrogenase (GAPDH; 1:50; sc-365062) primary antibodies as well as rabbit anti-mouse horseradish peroxidase-conjugated secondary antibodies (1:5,000; sc-358914) were obtained from Santa Cruz Biotechnology, Inc., (Santa Cruz, CA, USA).

Cell culture. All protocols in the present study were approved by the Ethics Committee of Guangxi Medical University, (Nanning, China). VSMCs were isolated from the thoracic aortas of 8 week-old male Sprague-Dawley rats, and cultured in DMEM/F12 medium with $10 \% \mathrm{FBS}$ at $37^{\circ} \mathrm{C}$ in a humidified atmosphere of $5 \% \mathrm{CO}_{2}$. VSMCs of Passage 3 were used in the present study.

Cell proliferation assay. The VSMCs $\left(5 \times 10^{3}\right)$ were cultured to $70 \%$ confluence in DMEM/F12 medium with $10 \% \mathrm{FBS}$ at $37^{\circ} \mathrm{C}$ in a humidified atmosphere of $5 \% \mathrm{CO}_{2}$ and serum-starved for $24 \mathrm{~h}$ in a 96 -well plate. VSMCs in the control group were cultured without any treatment. VSMCs in the PDGF-BB group were treated with PDGF-BB (30 ng/ml) for 6, 12, 24 and $48 \mathrm{~h}$. VSMCs in PDGF-BB+ligustrazine group were treated with PDGF-BB $(30 \mathrm{ng} / \mathrm{ml})$ and ligustrazine $(10 \mu \mathrm{M})$ for 6,12 , 24 and $48 \mathrm{~h}$. An MTT assay was used to examine the viability of the cells. The medium was added to MTT $(0.5 \mu \mathrm{g} / \mathrm{ml})$ and the cells were then incubated for $3 \mathrm{~h}$. Subsequently, the medium was removed, and $100 \mu \mathrm{l}$ DMSO was added. The plate was gently rotated at $0.67 \mathrm{x}$ g for $20 \mathrm{~min}$ using a desk centrifuge (ST40; Thermo Fisher Scientific, Waltham, MA, USA) to dissolve the precipitate. The microplate reader (3550; Bio-Rad Laboratories, Inc., Hercules, CA, USA) was used to determine the absorbance at $570 \mathrm{~nm}$.
NO assay. An NO enzyme immunoassay kit (Nitrite/Nitrate Assay kit; Sigma-Aldrich) was used to determine the level of $\mathrm{NO}$ in the medium of the VSMCs following treatment with PDGF-BB $(30 \mathrm{ng} / \mathrm{ml})$, with or without ligustrazine $(10 \mu \mathrm{M})$, for $48 \mathrm{~h}$, according to the manufacturer's instructions.

cGMP production assay. A cGMP enzyme immunoassay kit (HitHunter ${ }^{\mathrm{TM}}$ cGMP Assay kit; GE Healthcare, Franklin, NJ, USA) was used to determine the production of cGMP in the VSMCs following treatment with PDGF-BB $(30 \mathrm{ng} / \mathrm{ml})$, with or without ligustrazine $(10 \mu \mathrm{M})$, for $48 \mathrm{~h}$, according to the manufacturer's instructions. The total protein level in each group was examined using a bicinchoninic acid (BCA) protein assay kit (Thermo Fisher Scientific), and the data was normalized according to the total protein level.

Cell migration assay. A Transwell assay was performed to determine the rate of cell migration. A 24-well modified Boyden chamber containing fibronectin-coated polycarbonate membranes (BD Biosciences, Franklin Lakes, NJ, USA) was used to determine the VSMC migration in each group. Briefly, the lower wells in each group were filled with DMEM, with or without PDGF-BB $(30 \mathrm{ng} / \mathrm{ml})$, in the presence or absence of ligustrazine $(10 \mu \mathrm{M})$. The cells $\left(1 \times 10^{5}\right)$ were seeded on the upper side of the membrane and cultured in DMEM/F12 medium for $24 \mathrm{~h}$ at $37^{\circ} \mathrm{C}$ with $5 \% \mathrm{CO}_{2}$. Subsequently, the VSMCs on the upper side of the membrane were carefully removed, while the VSMCs on the lower side were stained with Hoechst 33342 (Sigma-Aldrich). The stained VSMCs were then counted in five randomly selected squares per well under an inverted microscope (IX71; Olympus, Tokyo, Japan).

Western blotting assay. Western blot analysis was performed to examine the protein expression levels in each group. The cells $\left(1 \times 10^{7}\right)$ were lysed in cold radioimmunoprecipitation assay buffer (Sigma-Aldrich). The BCA protein assay kit (Thermo Fisher Scientific) was used to determine the protein concentration, according to the manufacturer's instructions. Subsequently, the protein was separated with $10 \%$ SDS-PAGE gel (Sigma-Aldrich) and transferred onto a polyvinylidene difluoride (PVDF) membrane (Sigma-Aldrich). The PVDF membrane was blocked with 5\% nonfat dried milk (Mengniu Dairy Co., Ltd, Beijing, China) in phosphate-buffered saline (PBS; Sigma-Aldrich) for $4 \mathrm{~h}$. Subsequently, the PVDF membrane was incubated with specific primary antibodies for $3 \mathrm{~h}$ at room temperature. Following washing with PBS three times, each time for $5 \mathrm{~min}$, the PVDF membrane was incubated with the appropriate secondary antibody. Following washing with PBS three times, each time for 5 min, an Pierce ECL Plus Western Blotting Substrate kit (Thermo Fisher Scientific) was used to detect the immune complexes on the PVDF membrane.

Statistical analysis. Data are expressed as the mean \pm standard deviation of three independent experiments and were processed using SPSS 17.0 statistical software (SPSS, Inc., Chicago, IL, USA). The differences between groups were evaluated using one-way analysis of variance. $\mathrm{P}<0.05$ was considered to indicate a statistically significant difference. 
A
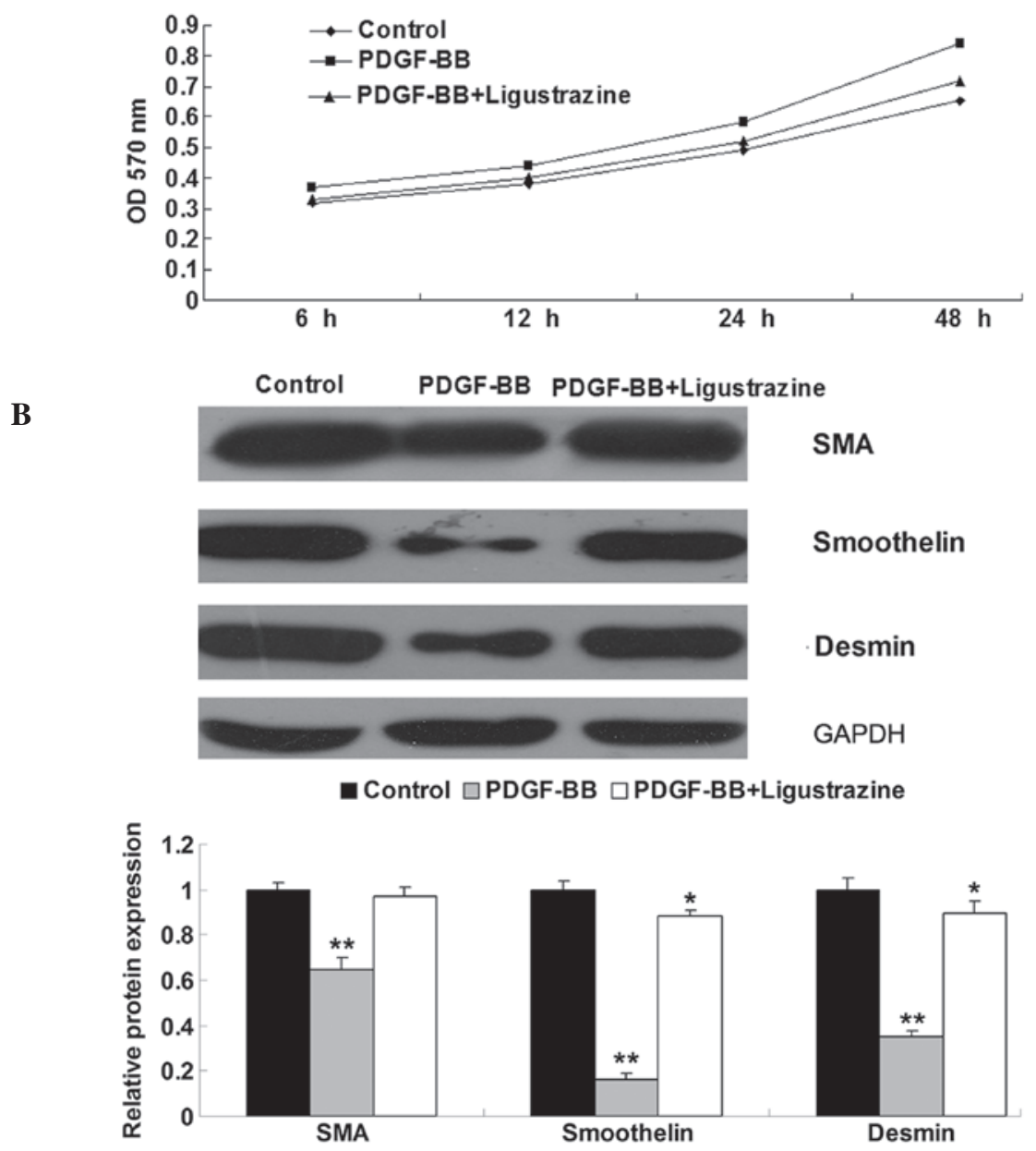

Figure 1. Ligustrazine inhibits PDGF-BB-induced VSMC proliferation. (A) An MTT assay was used to examine the effect of Ligustrazine and PDGF-BB on VSMC proliferation. Control VSMCs were cultured for 6, 12,24 and $48 \mathrm{~h}$ without any treatment. PDGF-BB VSMCs were treated with PDGF-BB for $6,12,24$ and 48 h. PDGF-BB+ligustrazine VSMCs were treated with ligustrazine and PDGF-BB for 6, 12, 24 and 48 h. (B) Protein expression levels of smooth muscle markers were determined by western blotting. Control VSMCs were cultured for $48 \mathrm{~h}$ without any treatment. PDGF-BB VSMCs were treated with PDGF-BB for $48 \mathrm{~h}$. PDGF-BB+ligustrazine VSMCs were treated with ligustrazine and PDGF-BB for $48 \mathrm{~h}$. Values are presented as the mean \pm standard deviation of three independent experiments. ${ }^{*} \mathrm{P}<0.05$ and ${ }^{* *} \mathrm{P}<0.01$ vs. control. VSMC, vascular smooth muscle cell; PDGF, platelet-derived growth factor; SMA, smooth muscle- $\alpha$-actin; OD, optical density.

\section{Results}

Ligustrazine inhibits PDGF-BB-induced VSMC proliferation. An MTT assay was initially performed to investigate the effects of PDGF-BB and ligustrazine on VSMC proliferation. As shown in Fig. 1A, the cell proliferation was significantly upregulated following treatment with PDGF-BB compared with the control group; however, ligustrazine effectively reversed the promoting effect of PDGF-BB on VSMC proliferation. Following vascular damage, VSMCs switch from a differentiated phenotype to a dedifferentiated phenotype and exhibit a higher proliferative ability. As SMA, smoothelin and desmin are three key markers for the differentiated VSMCs (1), western blot analysis was performed to determine the protein levels of these three markers. As shown in Fig. 1B, administration of PDGF-BB to the VSMCs significantly inhibited the protein expression of these three markers. Consistent with the aforementioned data from the MTT assay, these findings indicated that the VSMCs dedifferentiated into a proliferative phenotype. However, the expression of SMA, smoothelin and desmin in the VSMCs treated with PDGF-BB and ligustrazine were notably higher compared with those in the VSMCs treated with PDGF-BB alone. These findings suggested that ligustrazine inhibited the PDGF-BB-induced phenotype switch in the VSMCs.

Ligustrazine inhibits PDGF-BB-stimulated VSMC migration. A Transwell assay was used to further examine the effect of ligustrazine on PDGF-BB-stimulated VSMC migration. As shown in Fig. 2, VSMC migration was significantly upregulated following treatment with PDGF-BB for $48 \mathrm{~h}$, compared with that in the control group. However, ligustrazine effectively suppressed the PDGF-BB-induced upregulation of VSMCs migration.

Ligustrazine suppresses $P D G F-B B$-induced production of $N O$ and cGMP in VSMCs. The NO content of the supernatant and the production of cGMP in the VSMCs were further examined. As shown in Fig. 3A, the NO content in the supernatant of the VSMCs was significantly lower in the PDGF-BB group when compared with the control group. However, ligustrazine 


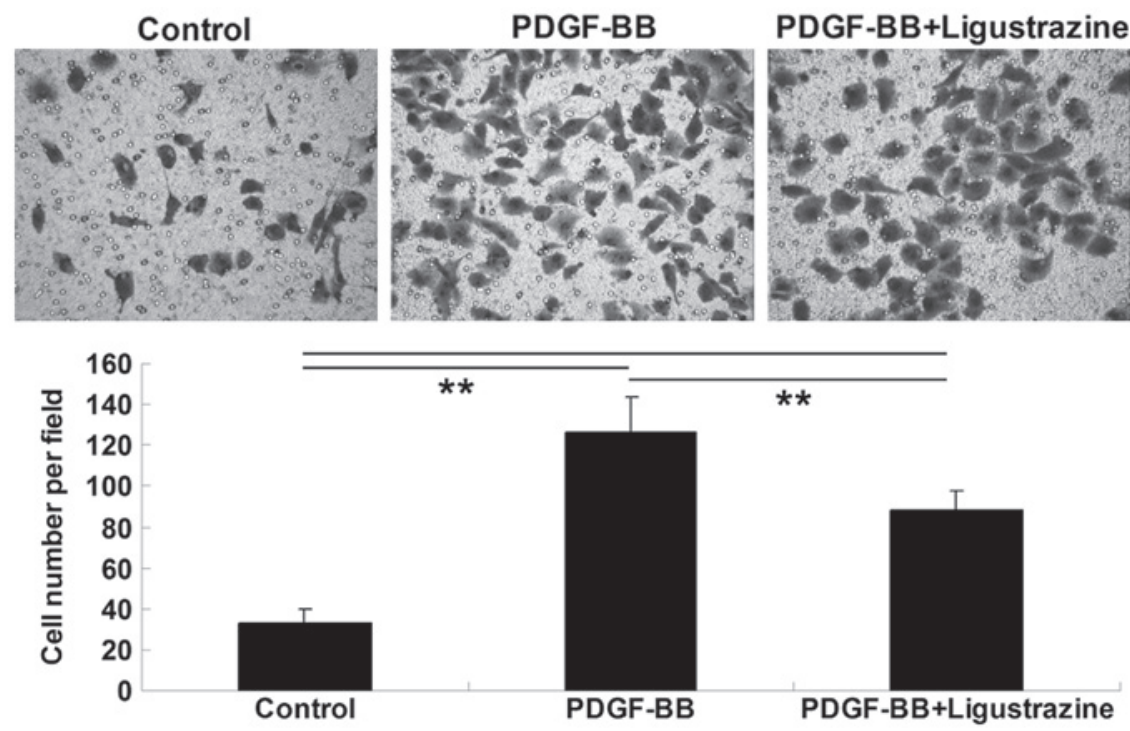

Figure 2. Ligustrazine suppresses PDGF-BB-induced VSMC migration. A Transwell assay was used to determine the effects of ligustrazine and PDGF-BB on VSMC migration. Control VSMCs were cultured for $48 \mathrm{~h}$ without any treatment. PDGF-BB VSMCs were treated only with PDGF-BB for $48 \mathrm{~h}$. PDGF-BB+ligustrazine VSMCs were treated with ligustrazine and PDGF-BB for $48 \mathrm{~h}$. The numbers of cells were counted in five randomly selected fields using an inverted microscope (magnification, $\mathrm{x} 100)$. Values are presented as the mean \pm standard deviation of three independent experiments $\left({ }^{* *} \mathrm{P}<0.01\right)$. VSMC, vascular smooth muscle cell; PDGF, platelet-derived growth factor.
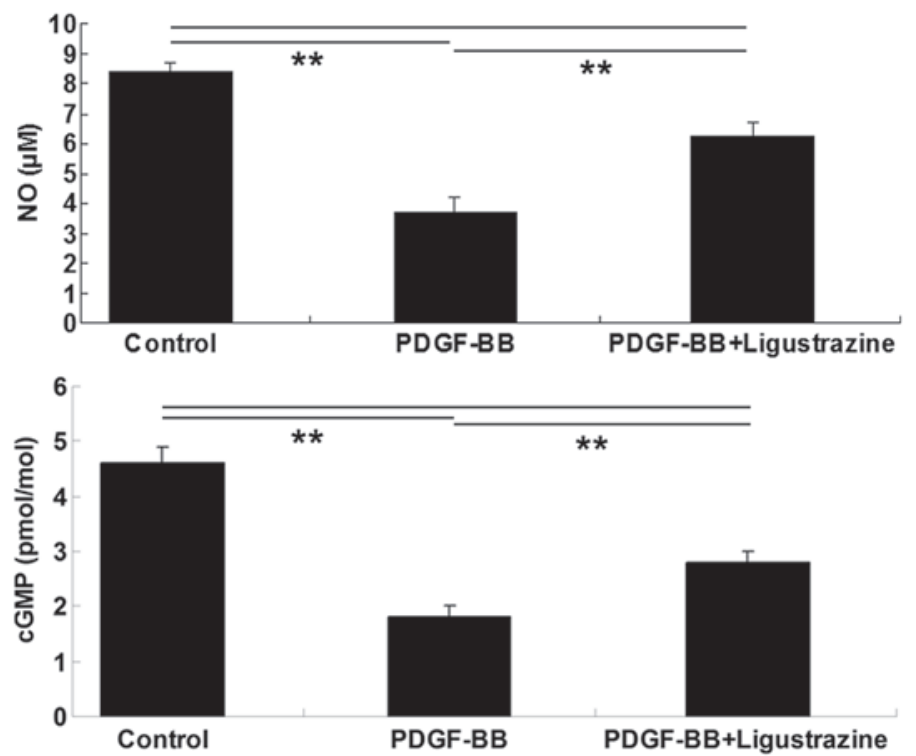

Figure 3. Ligustrazine suppresses the PDGF-BB-stimulated downregulation of NO and production of cGMP in VSMCs. The NO content in the medium and the cGMP level in the VSMCs were determined. Control VSMCs were cultured for $48 \mathrm{~h}$ without any treatment. PDGF-BB VSMCs were treated with PDGF-BB for $48 \mathrm{~h}$. PDGF-BB+ligustrazine VSMCs were treated with ligustrazine and PDGF-BB for $48 \mathrm{~h}$. Values are presented as the mean \pm standard deviation of three independent experiments ( ${ }^{* *} \mathrm{P}<0.01$ ). VSMC, vascular smooth muscle cell; PDGF, platelet-derived growth factor; NO, nitric oxide; cGMP, cyclic guanine monophosphate.

effectively reversed the PDGF-BB-induced downregulation of NO production. As shown in Fig. 3B, the production of cGMP was also decreased in the VSMCs following treatment with PDGF-BB for $48 \mathrm{~h}$, which was also reversed by the administration of ligustrazine.

Ligustrazine inhibits PDGF-BB-stimulated upregulation of cell cycle-associated proteins in VSMCs. Cyclins and CDKs have been demonstrated to act as key factors in the control of cell cycle progression, and their expression levels are mediated by
PDGF-BB in VSMCs $(14,15)$. To further investigate whether these proteins are involved in PDGF-BB- and ligustrazine-mediated VSMC proliferation, western blotting was used to examine the expression of cyclin D1, cyclin E, CDK2 and CDK4 in VSMCs treated with PDGF-BB, in the presence or absence of ligustrazine, respectively. As shown in Fig. 4, administration of PDGF-BB significantly upregulated the protein levels of cyclin D1, cyclin E, CDK2 and CDK4 in the VSMCs compared with the control group, and this was effectively reversed by treatment with ligustrazine, with the exception of CDK2. 

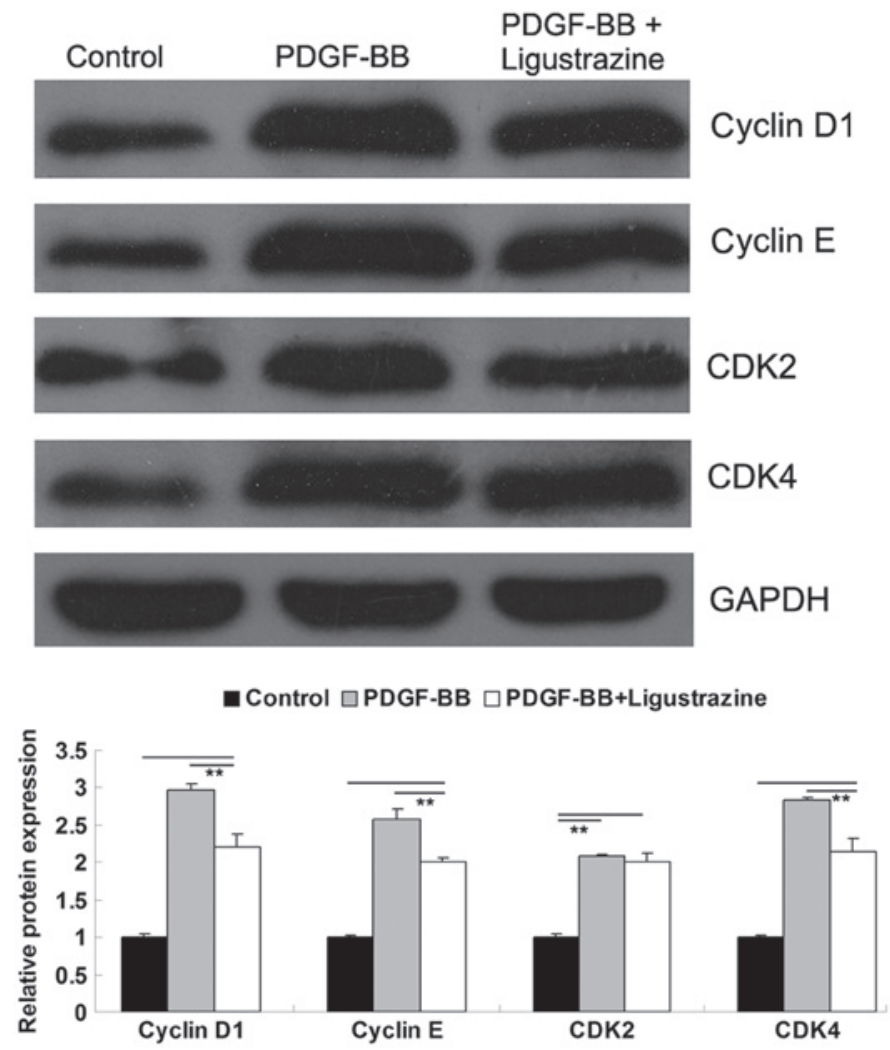

Figure 4. Ligustrazine suppresses the PDGF-BB-stimulated upregulation of cell cycle-associated protein levels in VSMCs. Western blotting was used to determine the levels of cell cycle related proteins, including cyclin D1, cyclin E, CDK2 and CDK4 in each group. Control VSMCs were cultured for $48 \mathrm{~h}$ without any treatment. PDGF-BB VSMCs were treated with PDGF-BB for $48 \mathrm{~h}$. PDGF-BB+ligustrazine VSMCs were treated with ligustrazine and PDGF-BB for $48 \mathrm{~h}$. Values are presented as the mean \pm standard deviation of three independent experiments ( $\left.{ }^{* *} \mathrm{P}<0.01\right)$. VSMC, vascular smooth muscle cell; PDGF, platelet-derived growth factor; CDK, cyclin-dependent kinase.

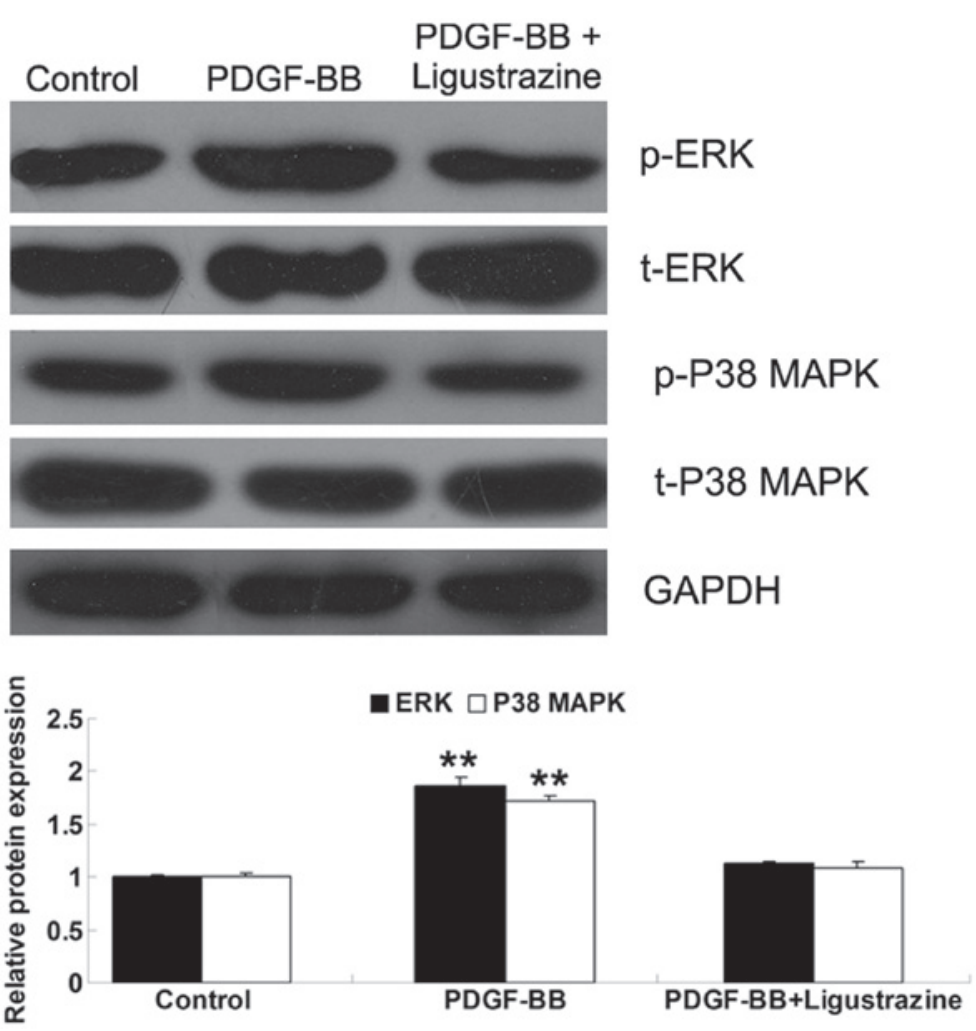

Figure 5. Ligustrazine suppresses PDGF-BB-stimulated activation of ERK and p38 MAPK signaling in VSMCs. Western blotting was used to determine the activity of ERK and p38 MAPK signaling in each group. Control VSMCs were cultured for $48 \mathrm{~h}$ without any treatment. PDGF-BB VSMCs were treated only with PDGF-BB for $48 \mathrm{~h}$. PDGF-BB+ligustrazine VSMCs were treated with ligustrazine and PDGF-BB for $48 \mathrm{~h} .{ }^{* *} \mathrm{P}<0.01$, vs. control. VSMC, vascular smooth muscle cell; PDGF, platelet-derived growth factor; ERK, extracellular signal-related kinase; MAPK, mitogen-activated protein kinase. 
Ligustrazine inhibits PDGF-BB-stimulated activation of ERK and P38 MAPK signaling in VSMCs. ERK and P38 MAPK signaling are important in the regulation of VSMC proliferation and migration. Accordingly, western blotting was used to examine the activity of ERK and P38 MAPK signaling in VSMCs treated with PDGF-BB, in the presence or absence of ligustrazine. As shown in Fig. 5, the expression levels of phospho-ERK and phospho-P38 MAPK in the VSMCs treated with PDGF-BB alone was notably upregulated compared with the control group. However, these effects were significantly reversed by the administration of ligustrazine. Therefore, it was suggested that ligustrazine had a suppressive effect on the PDGF-BB-induced activation of ERK and P38 MAPK signaling in the VSMCs, and that the inhibitory effect of ligustrazine on PDGF-BB-induced VSMC proliferation and migration was involved with its suppressive effect on the activation of ERK and P38 MAPK signaling.

\section{Discussion}

Following vascular injury, the production of inflammatory factors and cytokines, including PDGF-BB, is significantly increased, which has been observed to promote VSMC proliferation and migration and further cause neointima formation (16). In addition, neointima formation is closely associated with various cardiovascular diseases, including atherosclerosis and restenosis, following PCI $(1,2)$. As a result, the inhibition of cytokine-induced VSMC proliferation and migration offers promise for preventing the formation of neointima and certain cardiovascular disorders. Rhizoma Chuanxiong is among the most frequently used herbal medicinal products in China (17). Ligustrazine is its major constituent, which has a protective effect in cardiovascular diseases (4). However, whether ligustrazine has an effect on PDGF-BB-induced VSMC proliferation and migration and the molecular mechanisms involved remain to be elucidated. The results of the present study demonstrated for the first time, to the best of our knowledge, that ligustrazine effectively suppressed PDGF-BB-stimulated VSMC proliferation and migration, possibly through inhibition of their switch into a proliferative phenotype, cell cycle-related proteins upregulation, as well as ERK and P38 MAPK signaling activation.

In the present study, it was demonstrated, for the first time to the best of our knowledge, that ligustrazine suppressed PDGF-BB-induced VSMC proliferation. It has been well-established that VSMCs may dedifferentiate into a proliferative phenotype under proliferation-associated stimuli, including PDGF-BB treatment (18). Accordingly, the effect of ligustrazine on PDGF-BB-induced VSMC phenotype switch was further investigated and it was found that the administration of PDGF-BB significantly downregulated the protein levels of three VSMCs markers, SMA, Smoothelin and Desmin, suggesting that VSMCs dedifferentiated into a proliferative phenotype in response to PDGF-BB treatment. However, the treatment of ligustrazine restored their expression in VSMCs and maintained the differentiated phenotype. Consistent with the cell proliferation assay, these findings further confirmed that ligustrazine inhibited PDGF-BB-induced VSMC proliferation. Furthermore, as cyclin D1, cyclin E, CDK2, and CDK4 have been demonstrated to act as key factors in regulating cell cycle progression (14), their expression was further examined and it was demonstrated that administration of PDGF-BB significantly upregulated the protein levels of these cell cycle-related proteins in VSMCs, which was effectively reversed by treatment with ligustrazine, with the exception of CDK2. These findings suggested that the inhibition of cell cycle-related protein expression was involved in the suppressive effect of ligustrazine on PDGF-BB-induced VSMC proliferation.

However, it has also been demonstrated that VSMC migration is a key step during neointima formation and thus is involved in the development of atherosclerosis and restenosis following PCI (19). As a result, the roles of ligustrazine in the regulation of VSMC migration was further investigated, and it was found that treatment with PDGF-BB significantly promoted VSMC migration, which was effectively reversed by the administration of ligustrazine.

NO has been found to have a role in the regulation of the vascular system. For instance, it may inhibit platelet aggregation, leukocyte chemotaxis, as well as endothelial cell apoptosis $(20,21)$. In addition, NO has been revealed to suppress VSMC proliferation in vitro (22). NO may prevent the development of intimal hyperplasia following vascular injury (23). In addition, it has also been well-established that NO may stimulate the formation of cGMP, which also has a suppressive effect on VSMC proliferation (24). The present findings demonstrated that ligustrazine significantly inhibited PDGF-BB-induced downregulation of NO and cGMP production. These finding suggest that ligustrazine suppresses PDGF-BB-stimulated VSMC proliferation, partially at least, through regulating NO/cGMP-dependent mechanisms.

It has been well-documented that the ERK and the P38 MAPK signaling pathway in VSMCs is activated in response to treatment with PDGF-BB (1). In addition, the signaling is closely involved in the regulation of cell proliferation and migration (25). In addition, ERK and P38 MAPK signaling has been demonstrated to have a key regulatory role in vascular remodeling (26). Therefore, the present study further examined the activity of ERK and P38 MAPK signaling. The data revealed that ligustrazine inhibited PDGF-BB-induced ERK and P38 MAPK signaling activation, suggesting that the inhibitory role of ligustrazine in PDGF-BB induced VSMC proliferation and migration is through its repressive effect on the activation of the ERK and P38 MAPK signaling pathway.

In conclusion, the present study, for the first time to the best of our knowledge, demonstrated that ligustrazine downregulated PDGF-BB-stimulated proliferation and migration in VSMCs, partially at least, via its suppressive effects on PDGF-BB-induced ERK and P38 MAPK signaling activation and the expression of cell cycle related proteins. Accordingly, ligustrazine shows potential for the prevention and treatment of arteriosclerosis and restenosis following PCI.

\section{References}

1. Gan J, Li P, Wang Z, et al: Rosuvastatin suppresses platelet-derived growth factor-BB-induced vascular smooth muscle cell proliferation and migration via the MAPK signaling pathway. Exp Ther Med 6: 899-903, 2013. 
2. Lacolley P, Regnault V, Nicoletti A, Li Z and Michel JB: The vascular smooth muscle cell in arterial pathology: a cell that can take on multiple roles. Cardiovasc Res 95: 194-204, 2012.

3. Shi $\mathrm{N}$ and Chen SY: Mechanisms simultaneously regulate smooth muscle proliferation and differentiation. J Biomed Res 28: 40-46, 2014.

4. Zheng SD and Wu HJ: Progress of studies on effects of ligustrazine on blood vessel endothelium protection. Zhongguo Zhong Xi Yi Jie He Za Zhi 31: 1004-1008, 2011 (In Chinese).

5. Wang P, Luo CH, Wang QX, Li QY, Li P and Yuan XH: Antithrombotic effect of ligustrazine hydrochloride injection on the model of induced arteriovenous shunt thrombosis. J Res Med Sci 18: 704-706, 2013.

6. Luo ZL, Tian FZ, Tang LJ, et al: Protective effect of ligustrazine preconditioning on hepatic energy metabolism in ischemia/reperfusion injury of rat. Sichuan Da Xue Xue Bao Yi Xue Ban 43: 540-542, 2012 (In Chinese).

7. Yu Y, Wang SR and Sun YK: Effects of ligustrazine on the mitochondrial structure and functions in the process myocardial hypertrophy. Zhongguo Zhong Xi Yi Jie He Za Zhi 32: 661-665, 2012 (In Chinese)

8. Rocchiccioli S, Ucciferri N, Comelli L, Trivella MG, Citti L and Cecchettini A: Proteomics changes in adhesion molecules: a driving force for vascular smooth muscle cell phenotypic switch. Mol Biosyst 8: 1052-1059, 2012.

9. Orr AW, Hastings NE, Blackman BR and Wamhoff BR Complex regulation and function of the inflammatory smooth muscle cell phenotype in atherosclerosis. J Vasc Res 47: $168-180,2010$

10. Liu G, Li X, Li Y, et al: PPARdelta agonist GW501516 inhibits PDGF-stimulated pulmonary arterial smooth muscle cell function related to pathological vascular remodeling. Biomed Res Int 2013: 903947, 2013.

11. Park ES, Lee KP, Jung SH, et al: Compound K, an intestinal metabolite of ginsenosides, inhibits PDGF-BB-induced VSMC proliferation and migration through G1 arrest and attenuates neointimal hyperplasia after arterial injury. Atherosclerosis 228 53-60, 2013.

12. Conti V, Russomanno G, Corbi G, Izzo V, Vecchione C and Filippelli A: Adrenoreceptors and nitric oxide in the cardiovascular system. Front Physiol 4: 321, 2013.

13. Gibiino S1, Marsano A and Serretti A: Specificity profile of venlafaxine and sertraline in major depression: metaregression of double-blind, randomized clinical trials. Int J Neuropsychopharmacol 17: 1-8, 2014.

14. Lim S and Kaldis P: Cdks, cyclins and CKIs: roles beyond cell cycle regulation. Development 140: 3079-3093, 2013.
15. Sroka IM, Heiss EH, Havlicek L, et al: A novel roscovitine derivative potently induces G1-phase arrest in platelet-derived growth factor-BB-activated vascular smooth muscle cells. Mol Pharmacol 77: 255-261, 2010.

16. Pi Y, Zhang LL, Li BH, et al: Inhibition of reactive oxygen species generation attenuates TLR4-mediated proinflammatory and proliferative phenotype of vascular smooth muscle cells. Lab Invest 93: 880-887, 2013.

17. Zhang XL, Liu LF, Zhu LY, et al: A high performance liquid chromatography fingerprinting and ultra high performance liquid chromatography coupled with quadrupole time-of-flight mass spectrometry chemical profiling approach to rapidly find characteristic chemical markers for quality evaluation of dispensing granules, a case study on Chuanxiong Rhizoma. J Pharm Biomed Anal 88: 391-400, 2014

18. Fan Z, Li C, Qin C, et al: Role of the PI3K/AKT pathway in modulating cytoskeleton rearrangements and phenotype switching in rat pulmonary arterial vascular smooth muscle cells. DNA Cell Biol 33: 12-19, 2014

19. Karki R, Kim SB and Kim DW: Magnolol inhibits migration of vascular smooth muscle cells via cytoskeletal remodeling pathway to attenuate neointima formation. Exp Cell Res 319: 3238-3250, 2013.

20. Kobsar A, Klinker E, Kuhn S, et al: Increasing susceptibility of nitric oxide-mediated inhibitory platelet signaling during storage of apheresis-derived platelet concentrates. Transfusion 54: 1782-1789, 2014.

21. Qian J and Fulton D: Post-translational regulation of endothelial nitric oxide synthase in vascular endothelium. Front Physiol 4: 347,2013

22. Yoon $J$ and Ryoo S: Arginase inhibition reduces interleukin-1beta-stimulated vascular smooth muscle cell proliferation by increasing nitric oxide synthase-dependent nitric oxide production. Biochem Biophys Res Commun 435: 428-433, 2013.

23. Ataya B, Tzeng E and Zuckerbraun BS: Nitrite-generated nitric oxide to protect against intimal hyperplasia formation. Trends Cardiovasc Med 21: 157-162, 2011.

24. Yao M, Roberts DD and Isenberg JS: Thrombospondin-1 inhibition of vascular smooth muscle cell responses occurs via modulation of both cAMP and cGMP. Pharmacol Res 63: 13-22, 2011.

25. Kim EK and Choi EJ: Pathological roles of MAPK signaling pathways in human diseases. Biochim Biophys Acta 1802: 396-405, 2010

26. Muslin AJ: MAPK signalling in cardiovascular health and disease: molecular mechanisms and therapeutic targets. Clin Sci (Lond) 115: 203-218, 2008. 